Proceedings of the 1999 Particle Accelerator Conference, New York, 1999

\title{
THE MODEL OF ENSEMBLES FOR THE BEAM DYNAMICS SIMULATION *
}

\author{
A. Novokhatski ${ }^{\dagger}$, and T. Weiland, TEMF, TU Darmstadt
}

\section{Abstract}

It is proposed to use the "Ensembles" of particles for modeling the beams in accelerators. Each ensemble describes the longitudinal and transverse dynamics of the nonmonochromatic bunch in the six dimensional phase space, taking into account all coupling effects, coming from the relativistic relation between variation of the velocity projection and energy spread. Ensemble parameters include average values of coordinate and momentum, bunch dimensions, noncorrelated momentum spread and all second order correlation parameters. Self- consistent equations for the Ensemble are derived from the Vlasov equation. The presentation by vector and matrix gives the possibility to define an implicit algorithm for computer simulations. Examples of application of this model for the calculation of the beam dynamics in the accelerators are presented.

\section{INTRODUCTION}

In the beam dynamics calculations the beam is usually described by a set of "macro" particles. "Macro" particle is an ensemble of particles for the bunch field calculation. However for the trajectory calculation, a "macro" particle becomes a single particle. The motion of the particles inside "macro" particle is not considered. Therefore, the simulation of the beams with small emittance needs a relatively large number of 'macro" particles.

There is another possibility to describe the beam by the phase distribution function $f(\vec{r}, \vec{p})$ of particle density in the phase space of coordinates and momenta. Phase distribution function $f$ satisfies the Vlasov equation

$$
\frac{d}{d t} f=\frac{\partial f}{\partial t}+\overrightarrow{\operatorname{grad}_{r}}(f) \cdot \frac{\vec{p}}{\gamma} c+\overrightarrow{\operatorname{grad}_{p}}(f) \cdot \frac{\vec{F}}{m c}=0
$$

where normalized momentum $(\vec{p})$ and energy $(\gamma)$ are used

$$
\vec{p}=\frac{\vec{P}}{m c} \quad \gamma=\frac{E}{m c^{2}}=\sqrt{1+\vec{p} \bullet \vec{p}}
$$

and $\vec{F}$ is the force, acting on the particles $\frac{d}{d t} \vec{p}=\frac{\vec{F}}{m c}$. The direct numerical solution of the Vlasov equation in a 6 dimensional space needs a great amount of the computer memory. If we take only 50 mesh points for each direction, then the total number of mesh points will be $50^{6}=1.5610^{10}$, that is equal to the real number of particles in the bunch.

\footnotetext{
* Work supported in part by DESY, Hamburg

† Email: novot@temf.tu-darmstadt.de
}

\section{SELF-CONSISTENT BEAM MODEL}

However, the Vlasov equation can be numerically solved "much easily" for the linear forces. In this case the distribution function is described by a small number of parameters, only by the first and second order moments. Let us define such a bunch as an "Ensemble". If we have the full description of the dynamics of an Ensemble, then by a set of Ensembles we can describe the dynamics of any beam and all kinds of forces. Nevertheless, even one Ensemble can give a lot of information about beam dynamics in accelerator. Opposite to the usual model [1], [2], [3] for the second order moments, the model of Ensembles [4] includes longitudinal motion of the particles, and all corresponding correlations with transverse motion.

\subsection{Presentation by Vectors and Matrices}

The average coordinate and momentum of the Ensemble are described by vectors $\overline{\mathbf{X}}$ and $\overline{\mathbf{P}}$. The second order moments

$$
M_{\xi \nu}=\langle\xi \nu\rangle=\int f(t, \vec{r}, \vec{p})(\xi-\langle\xi\rangle)(\nu-\langle\nu\rangle) d \vec{r} d \vec{p}
$$

are grouped in matrices $\{\mathbf{S}\},\{\mathbf{L}\}$ and $\{\mathbf{T}\}$

$S_{i k}=M_{x_{i} x_{k}} \quad L_{i k}=M_{x_{i} p_{k}} \quad T_{i k}=M_{p_{i} p_{k}} \quad i, k=x, y, z$

The determinant of the matrix $\{\mathbf{M}\}$

$$
V^{2}=\operatorname{det}\{\mathbf{M}\}=\operatorname{det}\left(\begin{array}{cc}
\mathbf{S} & \mathbf{L} \\
\mathbf{L}^{\mathbf{t}} & \mathbf{T}
\end{array}\right)
$$

is the square of the 6 dimensional normalized emittance of the Ensemble. In the case of the uncoupling motions the full emittance is the multiplication of emittance projections

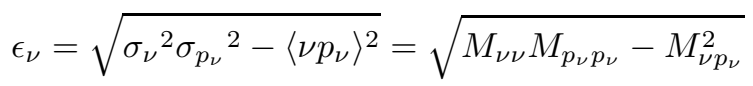

However, if the correlated moments appear, then the emittance projections are changed in order to keep the full emittance constant. For example, the full emittance is the difference of the positive values

$$
V^{2}=\epsilon_{y}^{2}\left(\epsilon_{x}^{2} \epsilon_{z}^{2}-M_{x p_{z}}^{2} \sigma_{p_{x}}^{2} \sigma_{x}^{2}\right)
$$

when the moment $M_{x p_{z}}$ is excited.

\subsection{Time Equations}

Time equations for the Ensemble parameters can be derived from the Vlasov equation under two assumptions:

1) If the applied forces satisfy the condition

$$
\left\langle\mu \bullet \overrightarrow{\operatorname{grad}_{p}} \frac{\vec{F}}{m c^{2}}\right\rangle=0
$$


for any Ensemble parameter $\mu$, then the full emittance is invariant.

2) The energy deviation in the beam is not very large and the energy can be presented in the following expanded way

$$
\begin{aligned}
\frac{1}{\gamma}=\frac{1}{\gamma_{m}}-\frac{1}{\gamma_{m}^{3}} \sum_{n}\left[\langle p _ { n } \rangle \left(p_{n}\right.\right. & \left.-\left\langle p_{n}\right\rangle\right)+ \\
& \left.+\frac{1}{2}\left(\left(p_{n}-\left\langle p_{n}\right\rangle\right)^{2}-M_{p_{n} p_{n}}\right)\right]
\end{aligned}
$$

where $\gamma_{m}$ is the average beam energy

$$
\gamma_{m}=\sqrt{1+\sum_{n}\left[\left\langle p_{n}\right\rangle^{2}+M_{p_{n} p_{n}}\right]}
$$

In this presentation the average velocity $\vec{v}$ contains additionally the momentum correlations

$$
v_{n}=\left\langle\frac{p_{n}}{\gamma}\right\rangle=\frac{\left\langle p_{n}\right\rangle}{\gamma_{m}}-\frac{1}{\gamma_{m}^{3}} \sum_{k}\left\langle p_{k}\right\rangle M_{p_{n} p_{k}}
$$

Under these conditions the Vlasov equation gives the formula for average value

$$
\frac{\partial}{c \partial t}\langle\mu\rangle=\left\langle\overrightarrow{g r a d_{r}} \mu \bullet \frac{\vec{p}}{\gamma}\right\rangle+\left\langle\overrightarrow{\operatorname{grad}_{p}} \mu \bullet \frac{\vec{F}}{m c^{2}}\right\rangle
$$

With this formula we present the time equations for moments in vector and matrix form:

$$
\begin{gathered}
\frac{\partial}{c \partial t} \overline{\mathbf{P}}=\mathbf{F} \\
\frac{\partial}{c \partial t} \overline{\mathbf{X}}=\frac{\mathbf{1}}{\gamma_{\mathbf{m}}}\left\{\mathbf{E}-\frac{\mathbf{1}}{\gamma_{\mathbf{m}}^{\mathbf{2}}} \mathbf{T}\right\} \otimes \overline{\mathbf{P}} \\
\frac{\partial}{c \partial t}\{\mathbf{S}\}=\{\mathbf{V}\} \otimes\left\{\mathbf{L}+\mathbf{L}^{\mathbf{t}}\right\} \\
\frac{\partial}{c \partial t}\{\mathbf{L}\}=\left\{\mathbf{V} \otimes \mathbf{T}+\mathbf{F}_{\mathbf{x}} \otimes \mathbf{S}+\mathbf{F}_{\mathbf{p}} \otimes \mathbf{L}\right\} \\
\frac{\partial}{c \partial t}\{\mathbf{T}\}=\left\{\mathbf{F}_{\mathbf{x}} \otimes \mathbf{L}+\mathbf{F}_{\mathbf{p}} \otimes \mathbf{T}\right\}+\left\{\mathbf{F}_{\mathbf{x}} \otimes \mathbf{L}+\mathbf{F}_{\mathbf{p}} \otimes \mathbf{T}\right\}^{\mathbf{t}}
\end{gathered}
$$

Matrix $\{\mathbf{V}\}$ is the symmetric matrix of combinations of velocity pairs $\left(v_{i}\right.$ and $\left.v_{k}\right)$

$$
V_{i k}=\frac{1}{\gamma_{m}}\left(\delta_{i k}-v_{i} v_{k}\right) \quad v_{k}=\frac{\overline{p_{k}}}{\gamma_{m}}
$$

$\delta_{i k}=1$, if $i=k$ and $\delta_{i k}=0$, if $i \neq k$

Vector $\mathbf{F}$ is the normalized average force

$$
\mathbf{F}=\frac{1}{m c^{2}} \mathbf{F}(\overline{\mathbf{X}}, \overline{\mathbf{P}})
$$

Matrices $\left\{\mathbf{F}_{\mathbf{x}}\right\}$ and $\left\{\mathbf{F}_{\mathbf{p}}\right\}$ are coordinate and momentum derivatives of the force

$$
F_{x}^{i k}=\left.\frac{1}{m c^{2}} \frac{\partial}{\partial x_{k}} F^{i}\right|_{\bar{X}, \bar{P}} \quad F_{p}^{i k}=\left.\frac{1}{m c^{2}} \frac{\partial}{\partial p_{k}} F^{i}\right|_{\bar{X}, \bar{P}}
$$

\section{PROPERTIES OF THE ENSEMBLE}

\subsection{Fundamental Conservation Laws}

We can check the model for realization of the dynamic laws for average values. It is easy to show, that from the presented above equations one obtains:

$$
\begin{gathered}
\frac{\partial}{c \partial t}\langle\gamma\rangle^{2}=\frac{\partial}{c \partial t} \gamma_{m}^{2}=2\left\langle\vec{p} \bullet \frac{\vec{F}}{m c^{2}}\right\rangle \\
\frac{\partial}{c \partial t} \overrightarrow{\mathcal{M}}=\frac{\partial}{c \partial t}\langle\vec{r} \times \vec{p}\rangle=\left\langle\vec{r} \times \frac{\vec{F}}{m c^{2}}\right\rangle
\end{gathered}
$$

So, the model fulfills the fundamental conservation laws for average momentum, energy and angular momentum.

\subsection{Emittance Equation}

We can also estimate the modification of the full emittance for the case, when the first assumption is not fulfilled. When we have only longitudinal force and transverse average momenta are zero, the model gives the following equation for longitudinal emittance

$\frac{\partial}{c \partial t} \epsilon_{z}^{2}=2 M_{z z}\left\langle\left(p_{z}-\left\langle p_{z}\right\rangle\right) \frac{F_{z}}{m c^{2}}\right\rangle-2 M_{z p_{z}}\left\langle(z-\langle x\rangle) \frac{F_{z}}{m c^{2}}\right\rangle$

When the force is $F_{z}=\alpha m c^{2} p_{z}$ (proportional to the momentum) the equation takes the form

$$
\frac{\partial}{c \partial t} \epsilon_{z}^{2}-2 \alpha \epsilon_{z}^{2}=0
$$

From this equation we get the exponential growth or damping of the emittance in time. The module of $1 / \alpha$ determines the effective time and the sign of $\alpha$ gives the type of the force: generator $(\alpha>0)$ or friction $(\alpha<0)$. We note, that at the same time the transverse emittances are invariant

$$
\frac{\partial}{c \partial t} \epsilon_{x}^{2}=0 \quad \frac{\partial}{c \partial t} \epsilon_{y}^{2}=0
$$

\subsection{Bunch Compression in Free Space}

Here we check the modification of the relativistic bunch in free space, when it has negative initial correlated momentum spread $M_{x p_{x}}^{0}$, or $M_{y p_{y}}^{0}$, or $M_{z p_{z}}^{0}$ If $\left\langle p_{z}\right\rangle \gg\left\langle p_{x}\right\rangle$, then

$$
\begin{aligned}
& M_{x x}=M_{x x}^{0}+2 \frac{c \Delta t}{\gamma_{m}} M_{x p_{x}}^{0}+\left(\frac{c \Delta t}{\gamma_{m}}\right)^{2} M_{p_{x} p_{x}}^{0} \\
& M_{z z}=M_{z z}^{0}+2 \frac{c \Delta t}{\gamma_{m}^{3}} M_{z p_{z}}^{0}+\left(\frac{c \Delta t}{\gamma_{m}^{3}}\right)^{2} M_{p_{z} p_{z}}^{0}
\end{aligned}
$$

The minimum size of the bunch is determined by the emittance and uncorrelated momentum spread

$$
\min \left(\sigma_{x}^{2}\right)=M_{x x}^{0}-\frac{\left(M_{x p_{x}}^{0}\right)^{2}}{M_{p_{x} p_{x}}^{0}}=\frac{\epsilon_{x}^{2}}{M_{p_{x} p_{x}}^{0}}=\frac{\epsilon_{x}^{2}}{\sigma_{p_{x}}^{2}}
$$

\section{APPLICATION OF THE MODEL}

The derived equations for the Ensemble parameters are nonlinear. So the numerical calculations need implicit algorithm. Nevertheless there are some analytical solutions for simple cases. We present examples of analytical and numerical solutions.

\subsection{The Ensemble in an Accelerating Structure}

Let the center of the bunch move with the speed equal to the phase velocity of the accelerating wave, having the phase displacement $\varphi_{0}$. Accelerating force is approximated by the linear part $\frac{F_{z}}{m c^{2}}=e+\delta E(z-\bar{z})$

$$
e=\frac{e}{m c^{2}} E_{a c c} \cos \varphi_{0} \quad \delta E=\frac{e}{m c^{2}} E_{a c c} \frac{2 \pi}{\lambda} \sin \varphi_{0}
$$

The model gives the following equations for $M_{z z}$ and $M_{z p_{z}}$

$$
\frac{\partial}{c \partial t} M_{z z}=\frac{2}{\gamma_{m}^{3}} M_{z p_{z}}
$$




$$
\frac{\partial}{c \partial t}\left(\gamma_{m}^{3} \frac{\partial}{c \partial t} M_{z p_{z}}\right)+4 \delta E M_{z p_{z}}=\delta E M_{z z} \frac{\partial \gamma_{m}^{3}}{c \partial t}
$$

From these equations we can estimate the frequency of the phase oscillations $\varpi$, when $\sin \varphi_{0}>0$

$$
\varpi / c=\sqrt{\frac{\delta E}{\gamma^{3}}}=\sqrt{\frac{2 \pi}{\lambda} \frac{e E_{a c c}}{\gamma^{3} m c^{2}} \sin \varphi_{0}}
$$

We've got exactly the M.Kapchinskiy formula [1].

\subsection{Space Charge Effect}

We use the estimation for the space charge force, derived out for the relativistic bunch of ellipse form, in the approximation of the homogenous charge density [4]

$$
\frac{\vec{F}}{m c^{2}}=\frac{r_{0} N}{\gamma^{2}} \frac{\vec{R}}{V_{g}}=æ \vec{R} \quad V_{g}=(\sqrt{5})^{3} \sqrt{\operatorname{det}\{\mathbf{S}\}}
$$

The force is linear with the distance $R$ and inversely proportional to the effective geometrical volume $V_{g} . N$ is the number of particles in the bunch, $r_{0}$ is the radius of the electron $r_{0}=e^{2} / m c^{2}$.

Our model gives the following equations for the transverse beam size $\sigma_{x}=\sqrt{M_{x x}}$ and correlated momentum spread $\sigma_{p_{x}}=\sqrt{M_{p_{x} x}}$

$$
\begin{gathered}
\frac{\partial}{c \partial t} M_{x x}=\frac{2}{\gamma_{m}} M_{x p_{x}} \\
\frac{\partial^{2}}{c^{2} \partial t^{2}} M_{x p_{x}}-\frac{4 æ}{\gamma_{m}} M_{x p_{x}}=0
\end{gathered}
$$

The effective distance $L_{\text {eff }}^{\perp}$, where the transverse beam size increases more than twice is

$$
L_{e f f}^{\perp}=\sqrt{\frac{\gamma_{m}}{æ}}=\sqrt{\frac{\gamma_{m}^{3} 5 \sqrt{5} \sigma_{x} \sigma_{y} \sigma_{z}}{r_{0} N}}
$$

One can find that this estimation is in very good agreement with the classical consideration [1], [3]. The effective parameter for the longitudinal size is $\gamma_{m}$ times larger.

\subsection{The Ensemble in the Magnetic Field}

A relativistic bunch with initial energy spread $\delta \gamma$ is injected into the magnetic field. Particles with different energy have different radiuses of rotation and therefore different time for one turn $T=\langle T\rangle \frac{\gamma}{\langle\gamma\rangle}$. $\langle T\rangle$ and $\langle\gamma\rangle$ are respectively the average period and energy. The bunch size $\sigma$ is

$$
\sigma=\langle R\rangle \sin \left(2 \pi \frac{t}{\langle T\rangle} \frac{\delta \gamma}{\langle\gamma\rangle}\right)
$$

After the time $T=\frac{\langle T\rangle}{4} \frac{\langle\gamma\rangle}{\delta \gamma}$ the bunch takes the circumference of the circle of the radius $\langle R\rangle$ Now we use numerical calculations to study this phenomena in the frame of our model. We have made calculations for the bunch with initial energy spread of $\pm 1 \%$. Computer results are shown on Fig.1. After 25 turns, the center of the bunch comes to the center of rotation and the beam size reaches the value of the rotation radius, the average momentum and its projections become zero, but the energy spread reaches maximum value. This position is repeated with the period of 50 turns.

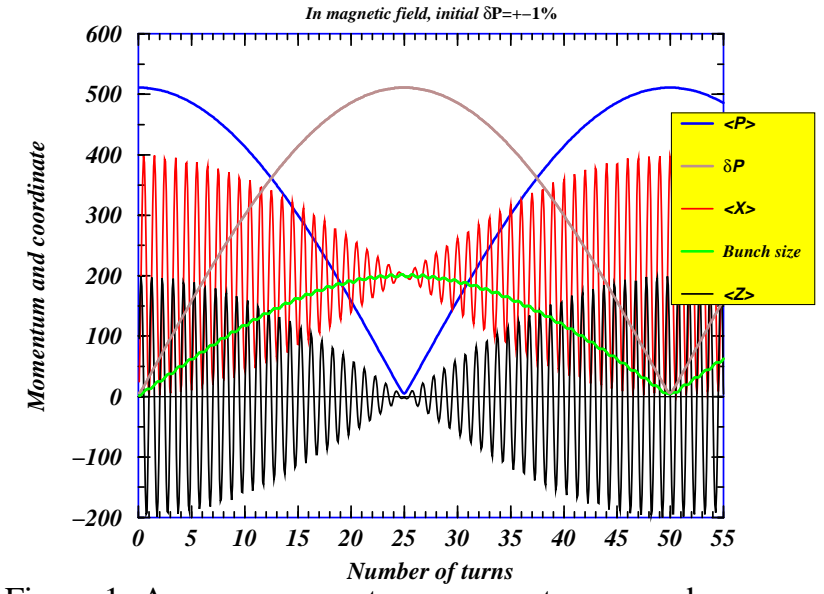

Figure 1: Average momentum, momentum spread, average $\mathrm{X}$-coordinate, bunch size and average Z-coordinate.

\subsection{Chicane Bunch Compression}

Chicane bunch compressor consists of four rectangular dipole magnets, where the bunch is deflected in transverse direction and then is forwarded back. When the bunch gets the transverse displacement $X$, it also gets correlated moments $M_{x p_{z}}, M_{z p_{x}}$ and $M_{p_{x} p_{z}}, M_{x z}$, that change the emittance projection

$$
\Delta \epsilon_{x}^{2}=\frac{X^{2}}{\gamma_{m}^{2}} M_{p_{z} p_{z}}^{0} M_{p_{x} p_{x}}^{0}
$$

In a symmetrical chicane the emittance projection comes back to the initial value. The space charge effect destroys the symmetry of the compression dynamics and increases the emittance projection. Results of computer simulations are presented on Fig.2.

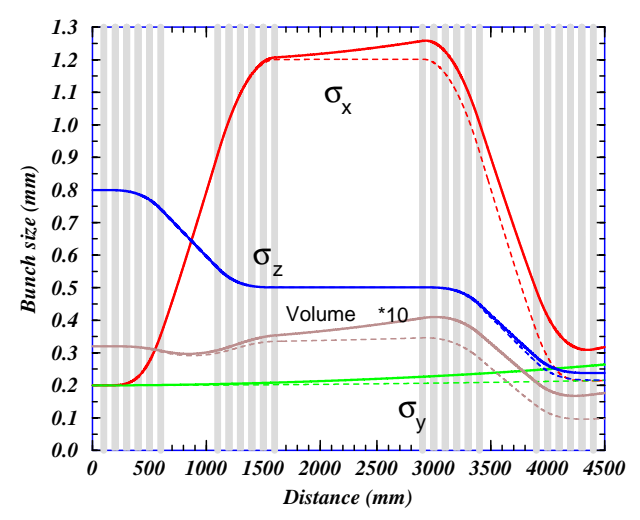

Figure 2: Bunch compression. The dashed lines show results without consideration of the space charge effect.

\section{REFERENCES}

[1] I.M.Kapchinskiy, Theory of Resonance Linear Accelerators, (Harwood Academic Publishers, Chur, 1985)

[2] A.Chao, Physics of Collective Beam Instabilities in High Energy Accelerators, (J.Wiley \& Sons, New York,1993)

[3] M.Reiser, Theory and Design of Charged Particle Beams, (J.Wiley \& Sons, New York,1994)

[4] A.N.Novokhatski and T.Weiland,'Self-consistent Model for the Beams in Accelerators", Proc. of ICAP, Monterey, 1998. 